\title{
LONG-TERM OUTCOME, SURVIVAL ANALYSIS, AND RISK STRATIFICATION OF DYNAMIC CARDIOMYOPLASTY
}

Anthony P. Furnary, MD ${ }^{a}$

Juan-Carlos Chachques, $\mathrm{MD}, \mathrm{PhD}^{\mathrm{b}}$

Luiz F. P. Moreira, MD $^{c}$

Gary L. Grunkemeier, $\mathrm{PhD}^{\mathrm{a}}$

Jeffrey $\mathrm{S}$. Swanson, $\mathrm{MD}^{\mathrm{a}}$

Noedir Stolf, $\mathrm{MD}^{\mathrm{c}}$

Sam Haydar, $\mathrm{MD}^{\mathrm{a}}$

Christoph Acar, MD

Albert Starr, MD $^{\mathrm{a}}$

Adib D. Jatene, $\mathrm{MD}^{\mathrm{c}}$

Alain F. Carpentier, MD, $\mathrm{PhD}^{\mathrm{b}}$
Methods: To analyze the long-term outcome of dynamic cardiomyoplasty, we retrospectively studied 127 consecutive patients who underwent this procedure in Paris, France $(n=76)$, São Paulo, Brazil $(n=37)$, and Portland, Oregon $(n=14)$. Preoperative data were collected for patients operated on between January 1985 and June 1994 and examined with respect to effect on long-term survival. Patients had a mean age of $50 \pm 13$ years and were predominantly male $(82 \%)$. In $46 \%$ the cause of disease was ischemic. Concomitant operations were performed in 22 patients. Results: Operative mortality was $12 \%(15 / 127)$. Kaplan-Meier survival \pm standard error at 1 through 5 years was $73 \% \pm 4 \%, 57 \% \pm 5 \%, 49 \% \pm 6 \%, 44 \% \pm 6 \%$, and $40 \%$ $\pm 7 \%$, respectively. There was a distinct improvement at 6 months in New York Heart Association functional class $(3.2 \pm 0.05$ vs $1.7 \pm 0.07, p<$ $0.0001)$ and a small but significant increase in left ventricular ejection fraction $(20 \% \pm 0.8 \%$ vs $23 \% \pm 1.5 \%, p=0.04)$. Ninety-day mortality was associated with low right ventricular ejection fraction, a blunted hemodynamic response to exercise testing, and requirement for an intraaortic balloon pump at the time of the operation. Using a stepwise Cox regression method of multivariable survival analysis $(n=101)$, we determined that atrial fibrillation, New York Heart Association class IV, high pulmonary capillary wedge pressure, and balloon pump use were independent variables simultaneously associated with poor overall survival. When metabolic testing variables were added to this model, peak oxygen consumption eliminated both pulmonary capillary wedge pressure and functional class from the model, albeit with fewer $(n=74)$ patients. Conclusion: Dynamic cardiomyoplasty is an evolving therapy for symptomatic congestive heart failure, the results of which may be enhanced by intelligent, risk-sensitive patient selection. (J Thorac Cardiovasc Surg 1996;112:1640-50)
D. ynamic cardiomyoplasty was first performed clinically in $1985 .^{1}$ It has since been used in the treatment of adult congestive heart failure in an estimated 600 patients worldwide. After the pio-

From Providence St. Vincent Hospital and Medical Center, Portland, Ore., ${ }^{\mathrm{a}}$ Hôpital Broussais, Paris, France, ${ }^{\mathrm{b}}$ and Instituto do Curacao, São Paulo, Brazil.

Read at the Twenty-first Annual Meeting of The Western Thoracic Surgical Association, Coeur d'Alene, Idaho, June 21-24, 1995

Received for publication June 21, 1995; revisions requested Oct. 25, 1995; revisions received June 17, 1996; accepted for publication June 18, 1996.

Address for reprints: Anthony P. Furnary, MD, Albert Starr Academic Center, 9155 SW Barnes Rd., Suite 240, Portland, OR 97225 .

Copyright (C) 1996 by Mosby-Year Book, Inc.

$0022-5223 / 96 \$ 5.00+0 \quad \mathbf{1 2 / 6 / 7 5 8 8 9}$ neering efforts of the Broussais Hospital group in Paris, the clinical application of dynamic cardiomyoplasty has expanded rapidly throughout Europe, South America, Canada, and the United States.

Outcomes analyses published to date have shown improvement in left ventricular ejection fraction, ${ }^{2-4}$ increases in stroke volume ${ }^{4}$ and stroke work index, ${ }^{3}$ dramatic improvements in New York Heart Association (NYHA) functional class, ${ }^{2-5}$ and improvement in cardiopulmonary oxygen use on exercise testing. A few reports to date ${ }^{6,7}$ have examined the factors associated with operative mortality. However, only one study ${ }^{8}$ has attempted to identify the preoperative factors that adversely affect long-term survival after dynamic cardiomyoplasty.

The purpose of this report was to produce a Cox hazard model that would identify which patients had a better chance of long-term survival after dynamic 
cardiomyoplasty. This was done by combining the long-term results of dynamic cardiomyoplasty from three major centers: The Hôpital Broussais, Paris, France; The Instituto do Curacao, São Paulo, Brazil; and St. Vincent Hospital, Portland, Oregon.

\section{Patients and methods}

Patient selection criteria. Patients selected for cardiomyoplasty were at high risk of dying within 1 year of severe cardiomyopathy. These included patients with NYHA class III or IV congestive heart failure (CHF) that was refractory to (i.e., did not improve despite) pharmacologic therapy, a left ventricular ejection fraction less than $40 \%$, left ventricular end-diastolic pressure or pulmonary capillary wedge pressure greater than $15 \mathrm{~mm} \mathrm{Hg}$, and an age between 18 and 80 years. Selected patients must be in stable enough condition to withstand the 12-week conditioning period required to transform the type II skeletal muscle fibers of the latissimus dorsi to type I, fatigue-resistant fibers capable of contracting up to 60 times per minute. ${ }^{9}$ As such, patients with arrhythmias necessitating an implantable device, vital capacity less than $55 \%$ of predicted, preoperative dependence on intravenous inotropic agents, life-threatening noncardiac disease, or those with previous cardiac operations were excluded.

The details of the surgical procedure have been well described $^{5,10}$ and will not be repeated here. Patients were evaluated before the operation and every 6 months by heart catheterization, radionuclide ventriculography, echocardiogram, and, when available, by cardiopulmonary exercise testing using a metabolic stress exercise protocol. Preoperative data were then examined with respect to their effect on short-term and long-term survival.

Statistical analysis. Data were collected separately at each of the three individual centers during the entire study period. In September 1994, on mutual agreement, these data were combined and collated to produce the analyses presented herein. Statistical Package for Social Sciences (SPSS) software (SPSS, Inc., Chicago, Ill.) was used to analyze all data. Results are reported as mean \pm standard error of the mean. Univariate comparisons were performed with two-tailed $t$ tests for continuous variables and Pearson's $\chi^{2}$ statistic for categorical variables. Significance levels were set at $p<0.05$. The Kaplan-Meier productlimit method ${ }^{11}$ was used to describe survival after cardiomyoplasty. A forward, variable selection, Cox proportional hazard model ${ }^{12}$ was used to evaluate the effect of risk factors on survival. To assess the fit of the resultant model, we plotted the logarithm of each patient's calculated hazard multiplier against his or her own actual survival to date.

\section{Results}

Patient population. Between January 1985 and June 1994, 127 patients meeting the selection criteria underwent dynamic cardiomyoplasty at The Hôpital Broussais, Paris, France $(n=76)$, Instituto de Curacao, São Paulo, Brazil $(n=37)$, and Provi- dence St. Vincent Hospital and Medical Center, Portland, Oregon $(n=14)$. Mean age was $50 \pm 13$ years and $82 \%$ of the population was male. Approximately half (58/127) had ischemic cardiomyopathy; in the remainder the etiology was classified as idiopathic. The mean NYHA class was $3.26 \pm 0.05$ (NYHA class IV, 33 patients; III, 89 patients; II, 2 patients; missing, 4 patients). A single patient underwent cardiomyoplasty with the right latissimus dorsi muscle, and 126 patients underwent left latissimus cardiomyoplasty in either the anterior or posterior positions. ${ }^{10}$ A concomitant cardiac procedure was performed on 22 patients: left ventricular aneurysm resection, 2 patients; coronary bypass procedure, 7 patients; mitral valve repair, 6 patients; left ventricular aneurysm repair plus coronary bypass, 2 patients; coronary bypass plus mitral valve repair, 1 patient; left ventricular aneurysm plus mitral valve repair, 1 patient; right ventricular tumor resection plus coronary bypass plus valve repair, 1 patient; and Fontan inferior vena cava-pulmonary artery valved conduit, 1 patient. Complete preoperative demographic, cardiac, exercise, and surgical data are presented in Table I.

Thirty-day operative mortality was $12 \%(15 / 127)$. Procedural mortality, defined as any death occurring up to 90 days after the operation, was $18 \%$ (20/113). Ninety days is believed to be a more clinically relevant cutoff for perioperative cardiomyoplasty mortality because it spans the 12-week skeletal muscle conditioning period required to achieve optimal cardiac augmentation.

A comparison of procedural survivors versus 90 day mortality is presented in Table II. Univariate analyses of patient-related variables revealed several potential factors associated with 90-day mortality. Significant differences are seen in both systolic and diastolic blood pressures at rest and during exercise. In addition, the increase in systolic blood pressure in response to exercise was significantly better in the procedural survivors, as was the peak oxygen consumption. The only surgical factor that was found to be associated with procedural mortality was the requirement for an intraaortic balloon pump (IABP) at the time of the operation. Poor right ventricular ejection fraction was also significantly associated with procedural mortality.

Procedural survivors had a significant increase in postoperative left ventricular ejection fraction at 6 months as compared with preoperative values (20 \pm 2 vs $23 \pm 2, p=0.04$ ). This improvement was sustained through 12 months (Fig. 1). The clinical 
Table I. Patient population: descriptive statistics

\begin{tabular}{|c|c|c|c|}
\hline Variable & Mean $\pm S D I$ & rentage & $n$ \\
\hline \multicolumn{4}{|l|}{ Demographics } \\
\hline Age (yr) & $50 \pm 13$ & & 127 \\
\hline Male sex & & 82 & 127 \\
\hline Ischemic cause & & 48 & 127 \\
\hline NYHA class & $3.3 \pm 0.5$ & & 123 \\
\hline \multicolumn{4}{|l|}{ Cardiac parameters } \\
\hline Atrial fibrillation & & 33 & 127 \\
\hline Cardiac index $\left(\mathrm{L} / \mathrm{min} / \mathrm{m}^{2}\right)$ & $2.3 \pm 0.6$ & & 104 \\
\hline Cardiothoracic ratio (CXR) & $0.56 \pm 0.07$ & & 120 \\
\hline End-diastolic diameter (mm) & $71 \pm 10$ & & 72 \\
\hline $\operatorname{EDVI}\left(\mathrm{ml} / \mathrm{m}^{2}\right)$ & $153 \pm 82$ & & 15 \\
\hline LVEDP (mm Hg) & $23 \pm 10$ & & 102 \\
\hline $\operatorname{LVEF}(\%)$ & $20 \pm 10$ & & 119 \\
\hline LVSWI ( $\mathrm{gm} / \mathrm{m}^{2}$ per beat) & $24 \pm 12$ & & 93 \\
\hline PAP mean (mm Hg) & $31 \pm 14$ & & 109 \\
\hline PCWP $(\mathrm{mm} \mathrm{Hg})$ & $22 \pm 10$ & & 106 \\
\hline PVCs per hour & $38 \pm 143$ & & 91 \\
\hline PVR (dynes) & $227 \pm 180$ & & 99 \\
\hline RVEF (\%) & $26 \pm 10$ & & 51 \\
\hline RVSWI (gm/ $\mathrm{m}^{2}$ per beat) & $7.8 \pm 4$ & & 92 \\
\hline \multicolumn{4}{|l|}{ Exercise testing } \\
\hline Heart rate-rest (beats/min) & $95 \pm 17$ & & 79 \\
\hline Heart rate-exercise (beats/min) & $143 \pm 24$ & & 79 \\
\hline Systolic BP-rest $(\mathrm{mm} \mathrm{Hg})$ & $114 \pm 20$ & & 77 \\
\hline Systolic BP_exercise $(\mathrm{mm} \mathrm{Hg})$ & $136 \pm 30$ & & 79 \\
\hline Diastolic BP-rest $(\mathrm{mm} \mathrm{Hg})$ & $75 \pm 12$ & & 76 \\
\hline Diastolic $\mathrm{BP}$ - exercise $(\mathrm{mm} \mathrm{Hg})$ & $83 \pm 15$ & & 77 \\
\hline $\mathrm{RPP}$ —rest (beats · mm Hg/min) & $108 \pm 25$ & & 76 \\
\hline $\mathrm{RPP}$ - exercise (beats $\cdot \mathrm{mm} \mathrm{Hg} / \mathrm{min}$ ) & $195 \pm 57$ & & 79 \\
\hline METS - rest & $1.1 \pm 0.3$ & & 75 \\
\hline METS-exercise & $4.5 \pm 1.6$ & & 79 \\
\hline $\mathrm{VO}_{2}$-rest & $343 \pm 132$ & & 76 \\
\hline $\mathrm{VO}_{2}$ - exercise & $1358 \pm 537$ & & 79 \\
\hline \multicolumn{4}{|l|}{ Surgical factors } \\
\hline Cardiopulmonary bypass used & & 22 & 126 \\
\hline Concomitant procedure done & & 22 & 127 \\
\hline IABP pump used & & 28 & 127 \\
\hline Left ventricular coverage (\%) & $90 \pm 2$ & & 85 \\
\hline Right ventricular coverage (\%) & $70 \pm 3$ & & 85 \\
\hline Patch required to complete wrap & & 50 & 104 \\
\hline
\end{tabular}

$n$, Number of patients for whom the variable is available; $S D$, standard deviation; NYHA, New York Heart Association; CXR, chest x-ray film; $E D V I$, end-diastolic volume index; $L V E D P$, left ventricular end-diastolic pressure; $L V E F$, left ventricular ejection fraction; $L V S W I$, left ventricular stroke work index; PAP, pulmonary artery pressure; $P C W P$, pulmonary capillary wedge pressure; $P V C$, premature ventricular contraction; $P V R$, pulmonary vascular resistance: $R V E F$, right ventricular ejection fraction; $R V S W I$, right ventricular stroke work index; $B P$, blood pressure; $R P P$, rate pressure product; $M E T S$, metabolic equivalents; $\mathrm{VO}_{2}$ maximum oxygen consumption; $L A B P$, intraaortic balloon pump.

relevance of these small but statistically significant objective changes is seen in dramatic improvements in NYHA class (Fig. 2) at both 6 and 12 months. Mean NYHA class for the group improved from $3.2 \pm 0.05$ to $1.6 \pm 0.6(p<0.0001)$ at 6 months and remained stable through 12 months as well.
A Kaplan-Meier survival curve for all patients is shown in Fig. 3. Three patients were lost to followup. Cumulative patient follow-up totaled 204 patient-years with an average of $1.7 \pm 0.1$ years per patient (including procedural deaths). This KaplanMeier analysis portrays survivals of $73 \% \pm 4 \%, 57 \%$ $\pm 5 \%, 49 \% \pm 6 \%, 44 \% \pm 6 \%$, and $40 \% \pm 7 \%$ at postprocedure years 1 through 5 , respectively. Causes of death included ventricular failure $(n=$ 26), sudden death $(n=23)$, pulmonary embolus $(n=2)$, stroke $(n=1)$, and sepsis $(n=1)$. There was a significant difference in the causes of procedural versus long-term mortality. Procedural mortality was mostly due to ventricular pump failure $(15 / 18=79 \%)$. Long-term mortality was mainly (48\%) due to arrhythmias, with progressive CHF causing only $42 \%(13 / 28)$ of the late deaths $(p=$ 0.01 vs procedural death caused by pump failure).

Preoperative demographic $(n=5)$, cardiac catheterization $(n=14)$, metabolic exercise testing $(n=$ $18)$, and surgical $(n=6)$ variables were first univariately compared (Table III). Univariately significant variables were then tested against overall survival in a series of multivariable Cox regression analyses to determine which factors play a simultaneous role in predicting the proportional hazard for long-term survival. This series of tests began with many variables and few patients. The least significant variables at the end of each run were progressively excluded to gain a larger sample of patients with complete data sets available for inclusion in the model. Once a model was finalized using only demographic, cardiac, and surgical variables (Cox model 1, Table III), cardiopulmonary metabolic exercise testing variables were then entered into the equation. This method significantly dropped the overall sample size (Cox model 2, Table III) but produced a distinct, but equally valid, model incorporating preoperative oxygen consumption measurements in the equation.

Cox regression model 1 included 101 of the 127 cases $(80 \%)$ and revealed that atrial fibrillation, elevated pulmonary capillary wedge pressure, NYHA class IV, and intraoperative requirement for IABP counterpulsation were all independent predictors of poor long-term survival.

Inclusion of the cardiopulmonary metabolic testing results, in addition to the variables analyzed in Cox regression model 1, decreased the valid sample size for the second model to 76 patients $(60 \%)$. In this model (Table III, Cox model 2), peak metabolic equivalents (METS) with exercise proved to be a 
Table II. Univariate analysis of 90-day procedural mortality

\begin{tabular}{|c|c|c|c|}
\hline Variable & Survivors & Deaths & $p$ Value \\
\hline \multicolumn{4}{|l|}{ Demographics } \\
\hline Age (yr) & $50 \pm 1$ & $51 \pm 4$ & 0.805 \\
\hline \multicolumn{4}{|l|}{ Center } \\
\hline $\mathrm{A}(\%)$ & 71 & 29 & \\
\hline $\mathrm{B}(\%)$ & 94 & 6 & 0.059 \\
\hline $\mathrm{C}(\%)$ & 78 & 22 & \\
\hline Male sex $(\%)$ & 82 & 85 & 0.730 \\
\hline Ischemic cause $(\%)$ & 44 & 65 & 0.091 \\
\hline NYHA class IV $(\%)$ & 24 & 45 & 0.094 \\
\hline \multicolumn{4}{|l|}{ Cardiac parameters } \\
\hline Atrial fibrillation $(\%)$ & 40 & 28 & 0.968 \\
\hline Cardiac index $\left(\mathrm{L} / \mathrm{min} / \mathrm{m}^{2}\right)$ & $2.3 \pm 0.06$ & $2.5 \pm 0.2$ & 0.408 \\
\hline Cardiothoracic ratio (CXR) (\%) & $56 \pm 0.6$ & $60 \pm 2$ & 0.068 \\
\hline End-diastolic diameter $(\mathrm{mm})$ & $72 \pm 1$ & $74 \pm 5$ & 0.361 \\
\hline $\operatorname{EDVI}\left(\mathrm{ml} / \mathrm{m}^{2}\right)$ & $134 \pm 18$ & $205 \pm 28$ & 0.083 \\
\hline LVEDP (mm Hg) & $22 \pm 1$ & $25 \pm 4$ & 0.342 \\
\hline $\operatorname{LVEF}(\%)$ & $20 \pm 1$ & $20 \pm 3$ & 0.851 \\
\hline LVSWI (gm/ $/ \mathrm{m}^{2}$ per beat) & $24 \pm 1$ & $22 \pm 4$ & 0.755 \\
\hline PAP mean $(\mathrm{mm} \mathrm{Hg})$ & $31 \pm 1$ & $33 \pm 3$ & 0.485 \\
\hline PCWP (mm Hg) & $21 \pm 1$ & $23 \pm 8$ & 0.400 \\
\hline PVCs per hour & $46 \pm 17$ & $19 \pm 18$ & 0.578 \\
\hline PVR (dynes) & $228 \pm 19$ & $214 \pm 53$ & 0.789 \\
\hline RVEF $(\%)$ & $27 \pm 1$ & $17 \pm 2$ & 0.015 \\
\hline RVSWI & $7.5 \pm 0.4$ & $9.4 \pm 1$ & 0.142 \\
\hline \multicolumn{4}{|l|}{ Exercise testing } \\
\hline Heart rate-rest (beats/min) & $95 \pm 2$ & $105 \pm 4$ & 0.055 \\
\hline Heart rate-exercise (beats/min) & $144 \pm 2$ & $154 \pm 9$ & 0.199 \\
\hline$\Delta$ Heart rate $(\%)$ & $55 \pm 3$ & $48 \pm 9$ & 0.457 \\
\hline Systolic BP-rest (mm Hg) & $115 \pm 2$ & $102 \pm 5$ & 0.028 \\
\hline Systolic BP-exercise $(\mathrm{mm} \mathrm{Hg})$ & $139 \pm 3$ & $110 \pm 5$ & 0.001 \\
\hline$\Delta$ Systolic BP $(\%)$ & $20 \pm 2$ & $8 \pm 4$ & 0.048 \\
\hline Diastolic BP-rest $(\mathrm{mm} \mathrm{Hg})$ & $76 \pm 1$ & $66 \pm 3$ & 0.006 \\
\hline Diastolic BP-exercise $(\mathrm{mm} \mathrm{Hg})$ & $85 \pm 2$ & $70 \pm 3$ & 0.003 \\
\hline$\Delta$ Diastolic BP $(\%)$ & $11 \pm 2$ & $6 \pm 4$ & 0.258 \\
\hline $\mathrm{RPP}$-rest (beats $\cdot \mathrm{mm} \mathrm{Hg} / \mathrm{min}$ ) & $109 \pm 3$ & $107 \pm 7$ & 0.836 \\
\hline $\mathrm{RPP}$ _exercise (beats $\cdot \mathrm{mm} \mathrm{Hg} / \mathrm{min}$ ) & $202 \pm 7$ & $167 \pm 9$ & 0.053 \\
\hline$\Delta \operatorname{RPP}(\%)$ & $89 \pm 7$ & $59 \pm 9$ & 0.100 \\
\hline METS-rest & $1.1 \pm 0.04$ & $1 \pm 0.07$ & 0.544 \\
\hline METS-exercise & $4.4 \pm 0.2$ & $3.7 \pm 0.5$ & 0.113 \\
\hline$\Delta$ METS $(\%)$ & $328 \pm 23$ & $274 \pm 6$ & 0.378 \\
\hline $\mathrm{VO}_{2}$-rest & $355 \pm 17$ & $286 \pm 24$ & 0.119 \\
\hline $\mathrm{VO}_{2}-$-exercise & $1409 \pm 62$ & $1039 \pm 193$ & 0.038 \\
\hline$\Delta \mathrm{VO}_{2}(\%)$ & $327 \pm 23$ & $265 \pm 53$ & 0.325 \\
\hline \multicolumn{4}{|l|}{ Surgical factors } \\
\hline Cardiopulmonary bypass used $(\%)$ & 18 & 40 & 0.097 \\
\hline Concomitant procedure done $(\%)$ & 80 & 70 & 0.354 \\
\hline IABP used $(\%)$ & 22 & 63 & 0.007 \\
\hline Left ventricular coverage (\%) & $91 \pm 2$ & $90 \pm 7$ & 0.910 \\
\hline Right ventricular coverage (\%) & $69 \pm 3$ & $75 \pm 8$ & 0.524 \\
\hline Patch required to complete wrap (\%) & 47 & 60 & 0.378 \\
\hline
\end{tabular}

The number of patients $(n)$ with valid data available for analysis is the same as that shown in table 1 for each variable. Comparison among the three centers was performed by a $\chi^{2}$ analysis (test statistic value $=5.67$, with 2 degrees of freedom). $N Y H A$, New York Heart Association; $C X R$, chest $\mathrm{x}$-ray film; $E D V$, end-diastolic volume index; $L V E D P$, left ventricular end-diastolic pressure; $L V E F$, left ventricular ejection fraction; $L V S W I$, left ventricular stroke work index; $P A P$, pulmonary artery pressure; $P C W P$, pulmonary capillary wedge pressure; $P V C$, premature ventricular contraction; $P V R$, pulmonary vascular resistance; $R V E F$, right ventricular ejection fraction; $R V S W$, right ventricular stroke work index; $B P$, blood pressure; $R P P$, rate pressure product; METS, metabolic equivalents; $\mathrm{VO}_{2}$ maximum oxygen consumption; $L A B P$, intraaortic balloon pump. 


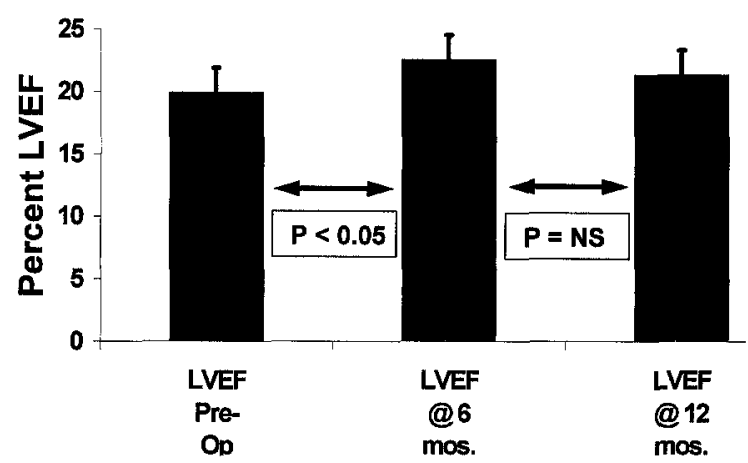

Fig. 1. Histogram comparing preoperative and 6 and 12 month postoperative left ventricular ejection fractions $(L V E F)$. Hatched bars represent one standard error of measurement. NS, Not significant.

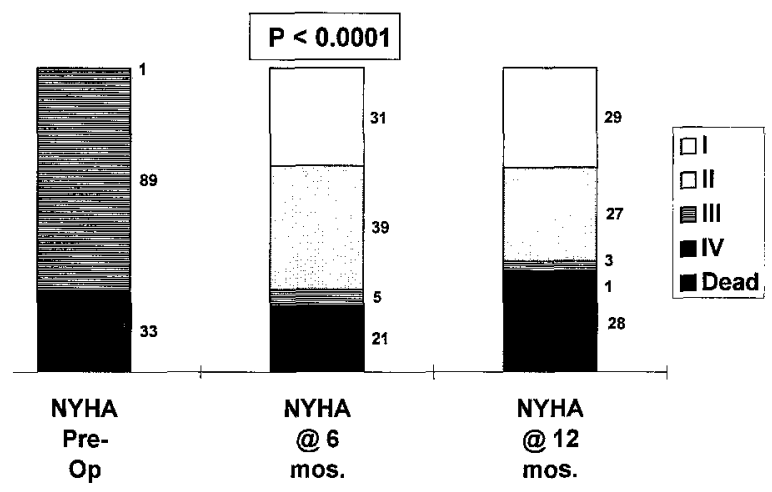

Fig. 2. Stacked histograms of mean NYHA functional class before the operation and 6 and 12 months after the operation.

strong surrogate for both pulmonary capillary wedge pressure and NYHA class, effectively eliminating these variables from the model. In this second model only peak METS, atrial fibrillation, and requirement for an IABP were significant predictors of a poor long-term outcome.

Using the larger Cox regression model 1 (without exercise testing parameters), we generated a composite risk score for each patient in the series and plotted it against that specific patient's actual survival to date. This method of internal validation is presented as a scatter diagram in Fig. 4.

\section{Discussion}

As one can see from the scatter diagram (Fig. 4), patients with high risk scores (above the composite risk $=0$ line) tend to die early, with very few of these patients remaining alive beyond

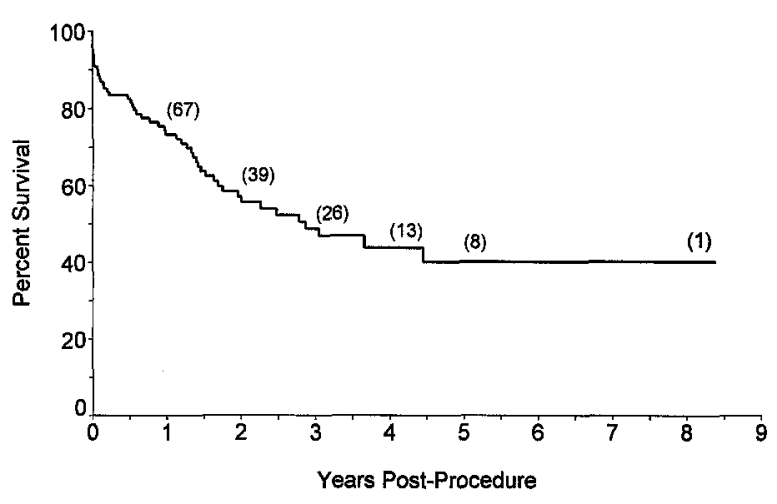

Fig. 3. Kaplan-Meier survival curve for 127 patients who underwent cardiomyoplasty between 1985 and 1994. Numbers above the curve represent the number of patients surviving to that point in time.

2 years. Patients with high composite risk scores tend to be grouped in the upper left-hand corner of the diagram, indicating a shorter overall survival. On the contrary, patients with composite risks less than 0 have a much lower likelihood of death at any time after the cardiomyoplasty procedure. Thus a much higher percentage of patients represented in the bottom half of the scatter diagram continue to survive long term.

This "composite risk score" generated from the Cox regression model enables one to estimate, for any patient in this series, the relative probability of a favorable long-term outcome. This is done by calculating the hazard multiplier for each patient, which is the exponentiated composite risk score. For example, when the composite risk score is 0 , the hazard multiplier is 1 . That is, the patient's hazard of dying is equal to that of the baseline hazard for the group. A negative composite score produces a hazard lower than the baseline hazard, whereas a positive score produces a hazard higher than the baseline hazard.

One must be careful not to extrapolate this specific Cox regression model to other groups of patients. This model was created on a specific set of patients and only internally validated. As such, it is not the predictive model for all centers in which cardiomyoplasty is performed. Because we did not have the luxury of extremely large sample sizes, only internal validation of the model could be performed. A more stringent test of this model would be to plot the composite risks of a large group of patients having cardiomyoplasty at other centers against their actual survival. External validation is the next logical step in determining a 
Table III. Multivariable Cox regression analyses

\begin{tabular}{|c|c|c|c|c|c|}
\hline \multirow[b]{2}{*}{ Variable } & \multirow[b]{2}{*}{ Univariate $p$ value } & \multicolumn{2}{|c|}{ Model $1(n=101)$} & \multicolumn{2}{|c|}{ Model $2(n=76)$} \\
\hline & & $p$ Value & Risk ratio & $p$ Value & Risk ratio \\
\hline \multicolumn{6}{|l|}{ Demographics } \\
\hline Age (yr) & 0.3 & & & & \\
\hline Center & 0.5 & & & & \\
\hline Male sex & 0.4 & & & & \\
\hline Ischemic cause & 0.5 & & & & \\
\hline NYHA class & 0.001 & 0.05 & $2.04(1.02,4.08)$ & & \\
\hline \multicolumn{6}{|l|}{ Cardiac parameters } \\
\hline Atrial fibrillation & 0.02 & 0.006 & $2.9(1.4,6.2)$ & 0.004 & $5.0(1.7,14.9)$ \\
\hline Cardiac index & 0.2 & & & & \\
\hline Cardiothoracic ratio & 0.05 & & & & \\
\hline LVEDD & 0.14 & & & & \\
\hline EDVI & 0.04 & & & & \\
\hline LVEDP & 0.2 & & & & \\
\hline LVEF & 0.4 & & & & \\
\hline LVSWI & 0.4 & & & & \\
\hline PAP mean & 0.02 & & & & \\
\hline PCWP & 0.01 & 0.01 & $1.05(1.01,1.09)$ & & \\
\hline PVCs per hour & 0.8 & & & & \\
\hline PVR & 0.02 & & & & \\
\hline RVEF & 0.3 & & & & \\
\hline RVSWI & 0.2 & & & & \\
\hline \multicolumn{6}{|l|}{ Exercise testing } \\
\hline Heart rate-rest & 0.2 & & & & \\
\hline Heart rate-exercise & 0.1 & & & & \\
\hline$\Delta$ Heart rate & 0.1 & & & & \\
\hline Systolic BP—rest & 0.07 & & & & \\
\hline Systolic BP-exercise & 0.02 & & & & \\
\hline$\Delta$ Systolic BP & 0.4 & & & & \\
\hline Diastolic BP-rest & 0.1 & & & & \\
\hline Diastolic BP-exercise & 0.2 & & & & \\
\hline$\Delta$ Diastolic BP & 1.0 & & & & \\
\hline RPP-rest & 0.5 & & & & \\
\hline $\mathrm{RPP}$ —exercise & 0.03 & & & & \\
\hline$\Delta \mathrm{RPP}$ & 0.1 & & & & \\
\hline METS-rest & 0.8 & & & & \\
\hline METS-exercise & 0.009 & & & 0.001 & $0.53(-37,0.78)$ \\
\hline$\Delta \mathrm{METS}$ & 0.03 & & & & \\
\hline $\mathrm{VO}_{2}$-rest & 0.4 & & & & \\
\hline $\mathrm{VO}_{2}$ - exercise & 0.03 & & & & \\
\hline$\Delta \mathrm{VO}_{2}$ & 0.05 & & & $*$ & \\
\hline \multicolumn{6}{|l|}{ Surgical factors } \\
\hline \multicolumn{6}{|l|}{ Cardiopulmonary bypass } \\
\hline Concomitant procedure & 0.9 & & & & \\
\hline IABP & 0.008 & 0.005 & $3.8(1.5,9.6)$ & 0.012 & $4.7(1.4,15.5)$ \\
\hline Left ventricular coverage & 0.7 & & & & \\
\hline Right ventricular coverage & 0.3 & & & & \\
\hline Patch to complete wrap & 0.8 & & & & \\
\hline Date of operation & 0.7 & & & & \\
\hline
\end{tabular}

Risk ratios are listed with $95 \%$ confidence intervals in parentheses. NYHA, New York Heart Association; $L V E D D$, left ventricular end-diastolic diameter; $E D V I$, end-diastolic volume index; $L V E D P$, left ventricular end-diastolic pressure; $L V E F$, left ventricular ejection fraction; $L V S W I$, left ventricular stroke work index; $P A P$, pulmonary artery pressure; $P C W P$, pulmonary capillary wedge pressure; $P V C$, premature ventricular contraction; $P V R$, pulmonary vascular resistance; $R V E F$, right ventricular ejection fraction; $R V S W I$, right ventricular stroke work index; $B P$, blood pressure; $R P P$, rate pressure product; $M E T S$, metabolic equivalents; $\mathrm{VO}_{2}$ maximum oxygen consumption; $I A B P$, Intraaortic balloon pump.

* $\mathrm{VO}_{2}$ is a surrogate covariate of METS (see Discussion). 


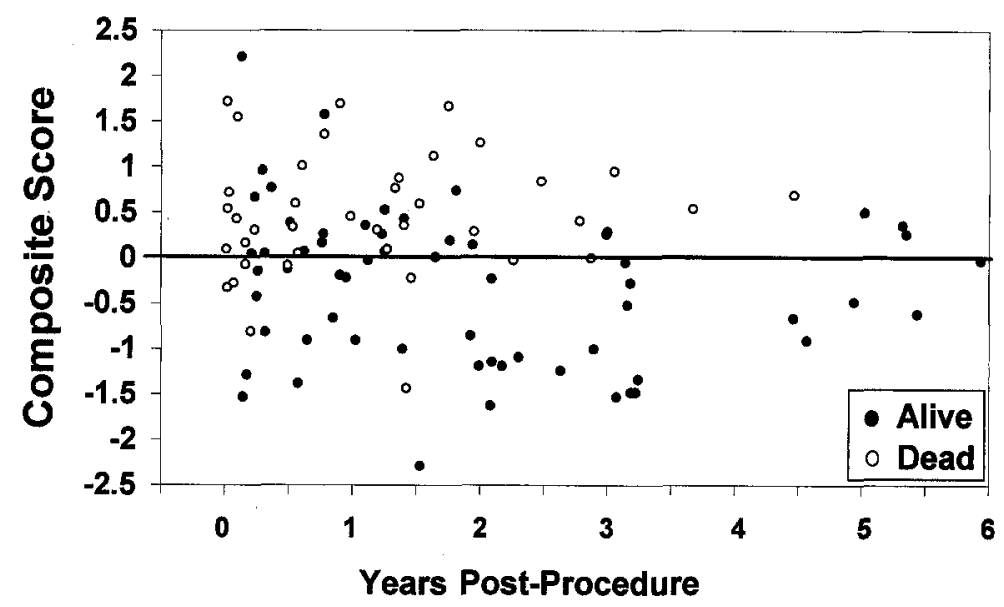

Fig. 4. Relationship of composite risk score generated from the Cox regression model (y-axis) to actual survival after cardiomyoplasty (x-axis).

universal risk stratification model of predictive survival for cardiomyoplasty.

This type of population risk stratification for overall long-term survival should be used to stratify the comparison of a group of patients having cardiomyoplasty with a control group of patients with CHF who are being medically treated. The risk stratification method of control obviates the need for specific matched controls between the groups. All variables found to be independent predictors of survival in this Cox regression have been previously shown to influence the natural history of medically treated patients with $\mathrm{CHF}$ as well. ${ }^{13-15}$

Atrial fibrillation has been shown to be an independent risk factor for early death in patients with CHF. ${ }^{13}$ Patients with CHF and atrial fibrillation have been found to have significantly lower actuarial survival and sudden death-free survival than similar patients with sinus rhythm. Atrial fibrillation as a risk factor might also apply to patients with seconddegree heart block or junctional rhythm, because loss of the atrial kick results in a $25 \%$ decrease in stroke volume. Other possible mechanisms for decreased survival caused by atrial fibrillation include an increased potential for thromboembolic events and an increased risk of ventricular tachyarrhythmias. ${ }^{14}$ There also exists the possibility that atrial fibrillation may be a marker for an immeasurable component of the severity and chronicity of left ventricular dysfunction.

Elevated pulmonary capillary wedge pressure has been shown to increase the risk of death in patients with CHF. ${ }^{13}, 15$ Likewise, increased pulmonary ar- tery diastolic pressure has also been shown to have a negative prognostic significance in medically treated patients with CHF. ${ }^{15}$ These two pulmonary pressure measurements tend to be highly dependent covariates. In this series elevation of pulmonary capillary wedge pressure proved to be a statistically stronger multivariate predictor of death, thereby eliminating pulmonary artery diastolic pressure from the final regression.

Peak oxygen consumption and its mathematical correlate, peak METS, are known to be codependent predictors of the risk of hemodynamic deterioration in patients with CHF. ${ }^{15}$ In this study, peak METS proved to be a statistically stronger variable. However, when peak METS was removed from the model, peak oxygen consumption (or alternatively, peak oxygen consumption per kilogram) immediately took its place in the equation with an equally strong predictive risk $(p=0.001, \beta$ coefficient $=$ 0.9983).

The requirement for IABP counterpulsation at the time of the operation greatly increases the risk of early death, as seen in Fig. 5, $A$. The effect of IABP use on long-term survival is not as dramatic as its effect on procedural mortality. When IABP use at the time of the operation was excluded from both multivariate models, no other variables came into the equation. Therefore IABP use did not mask any other variables available for analysis that might play an important role in long-term outcome. Although the requirement for an IABP at the time of cardiomyoplasty may be a risk that is difficult to avoid, it may also be a surrogate variable that signifies a 

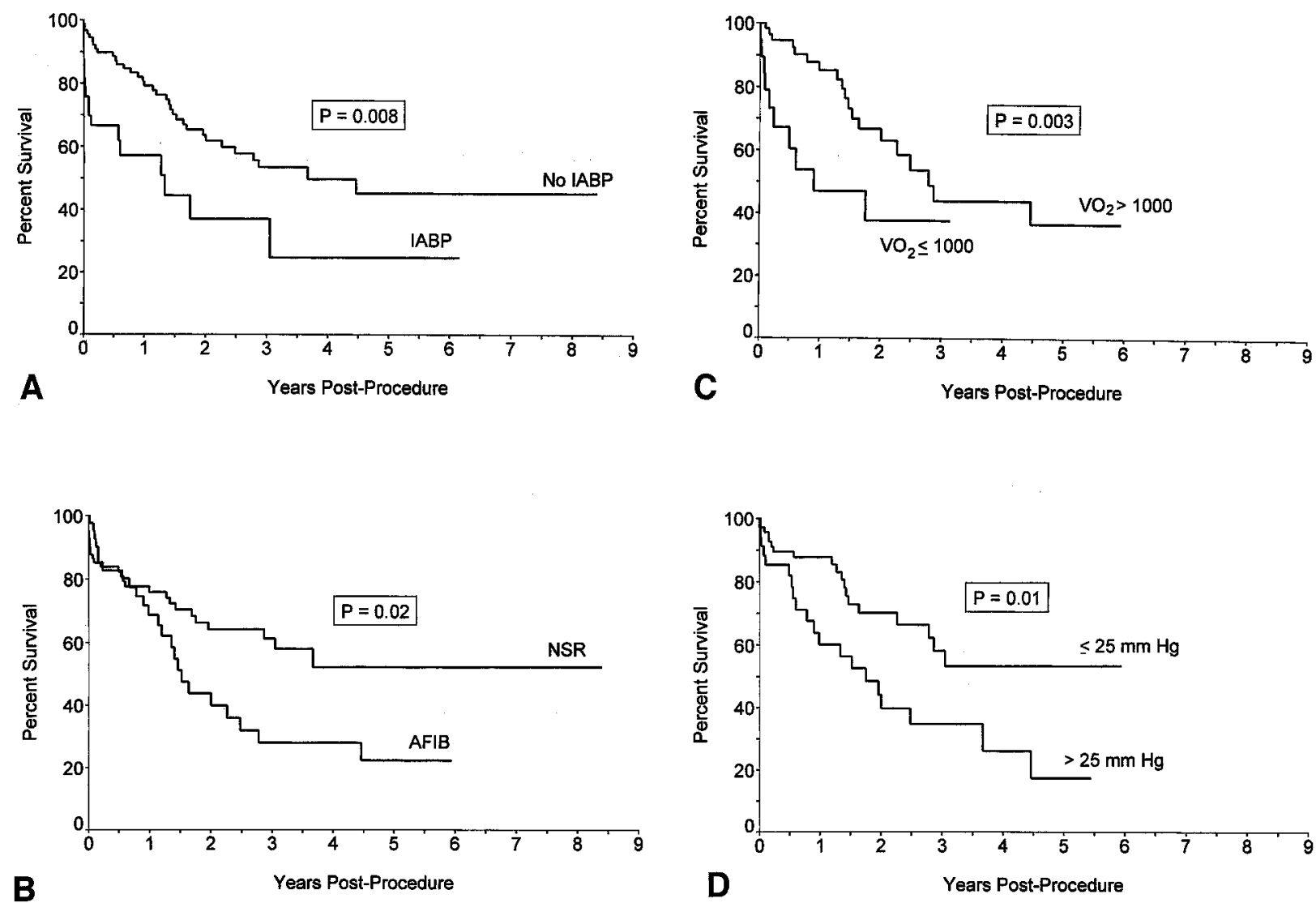

Fig. 5. Effect of four independent predictors of poor long-term survival that were identified by the Cox proportional hazards model. In each of these figures the survival of patients with and without the respective risk factor is compared. These predictors include IABP use (A), oxygen consumption $\left(\mathrm{VO}_{2}\right)(\mathbf{B})$, atrial fibrillation $(A F I B)(C)$, and pulmonary capillary wedge pressure (D). Note IABP and oxygen consumption effect on procedural deaths and effect of atrial fibrillation and pulmonary capillary wedge pressure on late deaths. The $p$ values are based on the log-rank test statistic. NSR, Normal sinus rhythm.

degree of myocardial damage and fibrosis beyond that which is indicated by traditional measures of left ventricular dysfunction.

The individual effect of each of these four independent predictors on long-term survival is illustrated in Fig. 5, $A$ to $D$. In each part of this figure, the Kaplan-Meier survival of patients with and without the respective risk factor is compared. As can be seen, all four parts show highly significant differences for each independent predictor of risk. For continuous variables, such as pulmonary capillary wedge pressure and maximum oxygen consumption, curves were generated for each patient group according to commonly accepted risk cutoffs. Close examination of Fig. 5, $A$ and $B$ reveal that intraoperative use of an IABP and a blunted hemodynamic response to preoperative exercise testing, as evidenced by a low peak oxygen consumption, are variables that greatly influence procedural mortality as opposed to long-term mortality. Conversely, atrial fibrillation and elevated pulmonary capillary wedge pressure have virtually no effect on procedural mortality, whereas they dramatically alter the late survival of patients who have undergone cardiomyoplasty. As such, the presence of atrial fibrillation or high pulmonary capillary wedge pressures should not influence surgeons to exclude patients from undergoing cardiomyoplasty, because they do not alter the immediate procedural risk. However, peak oxygen consumption and intraoperative use of an IABP do have procedural prognostic significance.

Inasmuch as cardiomyoplasty is currently being performed in nearly all areas of the globe, the importance in predicting survival, with and without intricate metabolic exercise testing, becomes obvious. Control patients who may not have undergone 
this expensive screening procedure may be easily compared with cardiomyoplasty patients who have undergone metabolic testing. Nonetheless, both Cox proportional hazard models are valid and should be applied to the appropriate groups. A blunted hemodynamic response to exercise testing may be a more cost-effective method of predicting a patient's procedural risk in centers where no complex metabolic testing equipment exists. Nonetheless, cardiopulmonary exercise testing remains the single best method to evaluate patients for risk of procedural mortality.

A multicenter group of 117 patients compiled by the Phase II Dynamic Cardiomyoplasty Study Group ${ }^{7}$ showed that although several factors were univariately associated with procedure-related death, only peak oxygen consumption and left ventricular ejection fraction provided independent prognostic information, according to a logistic regression analysis. In the present study, with a valid sample size of only one third of the total patient population, no consistent logistic regression analysis could be produced. Depending on the specific sample, each of the following variables was found to be a single independent predictor of risk by means of multivariable regression analysis: right ventricular ejection fraction, IABP use, peak METS, or peak oxygen consumption.

Preoperative NYHA class, in and of itself, should not be used as an exclusionary criterion in screening patients for cardiomyoplasty when metabolic exercising test data are available. Oxygen utilization measurements, obtained through exercise testing, are far superior to subjective NYHA classification in assessing a patient's procedural risk. Cardiopulmonary metabolic testing is extremely objective and the results are reported as a finely graded continuous score indexed to the patient's body size. These highly sensitive measurements carry much more precision than a subjective NYHA class in predicting procedural outcome.

The traditional definition of 30-day operative mortality has been modified in this analysis to an expanded 90-day procedural mortality. The rationale for this expanded time frame is both theoretical and real. The cardiomyoplasty procedure involves both an operation at a specific point in time and a prolonged muscle conditioning period that eventually allows that patient to achieve a clinical benefit from the operation. Unlike other surgical procedures, the patient does not immediately benefit from cardiomyoplasty within the first 30 days after operation. Skeletal muscle conversion to type I fibers capable of fatigue-resistant cardiac augmentation takes a full 12 weeks. ${ }^{9}$ Thus patients who die within 90 days of the procedure cannot clinically benefit from the procedure. In addition, mathematical analysis of the Kaplan-Meier survival curve (see Fig. 3) reveals a steep procedural mortality that extends to 3 months. This is then followed by a prolonged survival curve in which the death rate is markedly diminished. A linear fit to these two portions of the survival curve intersects at 90 days. For these reasons we thought that the 90-day procedural mortality end point for cardiomyoplasty was a more valid clinical and scientific concept.

\section{Summary}

This compiled multicenter study of 127 patients undergoing cardiomyoplasty reveals an acceptable long-term mortality that is altered by naturally occurring risks in patients with CHF. A 90-day procedural mortality is specifically appropriate to the cardiomyoplasty procedure in both the theoretical and the scientific sense. This procedural mortality is seen to be affected by low preoperative oxygen consumption on exercise testing and by the intraoperative requirement for IABP counterpulsation. Patients in NYHA class IV should not be excluded during the screening process when more objective and detailed peak oxygen consumption data are available. Likewise, atrial fibrillation and high pulmonary capillary wedge pressures should not be used to specifically exclude patients from the procedure, because these variables have an impact on late survival but do not seem to affect procedural mortality.

We conclude that the results of dynamic cardiomyoplasty for symptomatic CHF may be enhanced by intelligent, risk-sensitive patient selection. In addition, future outcomes comparison of patients with $\mathrm{CHF}$ treated either medically or by cardiomyoplasty should be performed on the basis of Cox risk stratification.

We greatly appreciate the kind and invaluable services of Andrew J Bennett, Natasha C Pfeifer, Cindy L Fessler, and Jennifer L. Bolger for assistance in preparation of the manuscript and figures.

REFERENCES

1. Carpentier A, Chachques JC. Myocardial substitution with a stimulated skeletal muscle: first successful clinical case [letter]. Lancet 1985;8440:1267.

2. Moriera LFP, Bocchi EA, Stolf NA, Pileggi F, Jatene AD. 
Current expectations in dynamic cardiomyoplasty. Ann Thorac Surg 1993;55:299-303.

3. Furnary AP, Moreira LFP, Jessup M. The American Cardiomyoplasty Group. Dynamic cardiomyoplasty improves systolic ventricular function. Circulation 1994:90-309.

4. Larusso R, Zogno M, LaCanna G, et al. Dynamic cardiomyoplasty as an effective therapy for dilated cardiomyopathy. $\mathrm{J}$ Cardiac Surg 1993;8:177-83.

5. Carpentier A, Chachques JC, Acar C, et al. Dynamic cardiomyoplasty at seven years. J Thorac Cardiovasc Surg 1993;106: 42-54.

6. Magovern JA, Furnary AP, Christlieb IY, Kao RL, Park SB, Magovern GJ. Indications and risk analysis for clinical cardiomyoplasty. Sem Thorac Cardiovase Surg 1991;3:145-8.

7. Phase II Dynamic Cardiomyoplasty Study Group. Factors associated with acute hospital mortality following a latissimus dorsi cardiomyoplasty. J Am Coll Cardiol 1994;859:1A-484A, \#859.2.

8. Furnary AP, Magovern JA, Swanson JS, et al. Pre-operative factors affecting survival after cardiomyoplasty: a multivariate analysis in cardiomyoplasty. In: Carpentier A, Chachques JC, editors. Mount Kisko (NY): Futura. In press.

9. Chiu RCJ. Using skeletal muscle for cardiac assistance. Sci Am Sci Med 1994;1:68-77.

10. Furnary AP, Christlieb IY, Magovern JA, Magovern GA. A standardized nomenclature for latissimus dorsi cardiomyoplasty. J Cardiac Surg 1991;6:74-9.

11. Kaplan EL, Meier P. Nonparametric estimation from incomplete observations. J Am Stat Assoc 1958;53:457-81.

12. Cox DR. Regression methods and life tables. J R Stat Soc 1972;34:187-220.

13. Middlekauff HR, Stevenson WG, Stevenson LW. Prognostic significance of atrial fibrillation in advanced heart failure: a study of 390 patients. Circulation 1991;84:40-8.

14. Gomes AJ, Alexopoulous D, Winters SL, Deshmukh P, Fuster V, Suh K. The role of silent ischemia, arrhythmic substrate and the short-long sequence in the genesis of sudden cardiac death. J Am Coll Cardiol 1989;14:1618-25.

15. Saxon LA, Stevenson WG, Middlekauff HR, et al. Predicting death from progressive heart failure secondary to ischemic or idiopathic dilated cardiomyopathy. Am J Cardiol 1993;72: 62-5.

\section{Discussion}

Dr. Richard J. Hurvitz (Los Angeles, Calif.). The concept of transforming a readily available skeletal muscle into functioning cardiac muscle and then positioning this muscle so as to augment failing heart muscle is truly an exciting concept. However, why then after more than 10 years since the concept first materialized is the procedure still controversial, particularly here in the United States? The coronary bypass graft as well as valve replacement both rapidly gained acceptance and proliferated in exponential fashion. Heart transplantation initially went through a skeptical phase but is now firmly established as an accepted treatment modality. There are estimated to be more than 2 million patients here in the United States with chronic CHF and an estimated 400,000 new cases each year. I believe the controversy over cardiomyoplasty has lingered for this relatively long period of time because the data to validate this procedure have not been convincing and the mechanism of action has not been well defined. I want to congratulate Dr. Furnary on his attempt to retrospectively retrieve information from three major centers performing cardiomyoplasty that ideally will shed some additional light on cardiomyoplasty. We can agree with Dr. Furnary's conclusion that cardiomyoplasty may be an effective form of therapy for patients with symptomatic CHF. However, additional data will be necessary to validate this. The Medtronic phase III study, which is a randomized trial comparing standard medical therapy for CHF versus standard medical therapy plus cardiomyoplasty, should help address this issue. This study recently got underway. Those of us who have followed cardiomyoplasty over the years are awaiting information that will either vindicate this procedure or inspire development of a modified procedure that will use the general concept of using the transformed skeletal muscle to assist the failing heart. Ideally, with regard to cardiomyoplasty, it will not be the case that when all is said and done, more will be said than done.

I have several questions for Dr. Furnary. First, what is your opinion of the mechanism of action of cardiomyoplasty?

Dr. Furnary. We believe there are three possible mechanisms for cardiomyoplasty. First is its effect on systolic function and augmentation thereof. At the phase II results from cardiomyoplasty, which were presented at the American Heart Association meeting, there was a significant increase in stroke volume and stroke work index. These were statistically and clinically significant.

The second theory is that dynamic cardiomyoplasty inhibits the progressive dilation of the ventricle during exercise, specifically submaximal exercise.

Finally there is the theory that electrical stimulation of a neuromuscular complex may alter the neurohumoral baseline status of the patient, such that there are increased levels of circulating catecholamines which may indirectly affect patient well-being and cardiac function.

Dr. Hurvitz. Which do you think is the dominant factor?

Dr. Furnary. The only scientifically proven dominant factor from the phase II study is systolic augmentation.

Dr. D. Craig Miller (Stanford, Calif.). What about diastole?

Dr. Furnary. There have been experimental results showing improvement in diastolic function; however, the application of the clinical mechanisms to evaluate diastolic function such as the use of conductance catheters has not been applied to this group of patients. It has only been applied in the laboratory, but there are very good laboratory results suggesting that there may be some improvement in diastolic function.

Moderator. I rather disagree with you there. There was a study from Belgium just a couple months ago showing that diastolic function was in fact the most predominant.

Dr. Furnary. Thank you. I missed that paper.

Dr. Hurvitz. Cardiomyoplasty has been labeled as an alternative for cardiac transplantation. In view of your conclusion, I believe that you state that patients in NYHA class IV should not be excluded from the procedure. Do you believe this procedure is an alternative for heart transplantation?

Dr. Furnary. I do not and I have not classified the 
procedure as an alternative to heart transplantation or a replacement thereof. I personally have positioned this procedure as an adjuvant to medical therapy for those patients who do not respond to medical therapy. For patients who are receiving maximum medical therapy, who are in class III or in early class IV CHF, and have not responded, this is an adjuvant procedure for medical therapy. We do not take patients off of their medical therapy, and certainly this is not meant to replace cardiac transplantation. In its present state I do not think it will. Twelve patients world wide have gone on to transplantation from cardiomyoplasty.

In terms of patients with NYHA class IV disease, I believe that exercise testing parameters for oxygen consumption are much more precise and are much more objective than NYHA class in assessing a patient's risk. However, not all centers around the world may have these expensive pieces of equipment available to them. They could fall back on the measurement of pulmonary pressures and NYHA subjective class to assess preoperative risk.

Dr. Hurvitz. How do you think the stance adopted by Medicare and some private insurance agencies on reimbursing for experimental devices will affect the progress of the project here in the United States?

Dr. Furnary. That is a complex question. Actually it ties in with what you said originally, because this is a controversial procedure. Part of the controversy relates to the fact that we still do not know whether this modifies or improves patient survival. We have seen subjective improvements in $\mathrm{CHF}$, but again that is a clinical controversial area.

There is also the controversial area of payment for experimental procedures. This is the first surgical procedure that is fully regulated by the Food and Drug Administration, and they have told us how it has to be done. None of the modifications that have come to bear over the past 5 or 6 years in the laboratory may be used or incorporated into the procedure. Also, in terms of the Food and Drug Administration and the Health Care Financing Administration, there remains the paradox. Although the FDA wants us to do this study and we want to do this study and find out whether it really does affect long-term mortality, Medicare will not pay for the procedure because it is experimental. It is not necessarily paying for the procedure but the hospital stay related to the procedure. The hospitals have to write off the entire cost of this procedure, and the only centers are actively performing this procedure in this country are centers that have large clinical endowments for clinical experimental surgery. Some of the centers have had large fund raisers specifically for this purpose, but insurance companies follow suit with Medicare. Until the paradox is resolved at the governmental level, the development of this procedure will stagnate in the United States. At the same time, the procedure is proliferating throughout Europe and Asia and South America.

Dr. Miller. Were these cardiomyoplasties all left posterior wraps? Were there any right anterior wraps?

Dr. Furnary. There were no right anterior wraps. There was one right posterior wrap for a patient with right ventricular failure, but otherwise this series comprised exclusively left ventricular wraps.

Dr. Noel H. Fishman (Santa Cruz, Calif.). Your repeated emphasis on the "statistically significant" but rather small (only 2\%) increase in left ventricular ejection fraction after cardiomyoplasty promotes the inference that you consider it an "important" determinant in the dramatic improvement in the NYHA classification of these patients. I find this hard to believe.

Clinical improvement is, of course, a subjective phenomenon and could have been related to other factors such as improved drug treatment regimens. Do you have any other physiologic data to support your hypothesis?

Dr. Furnary. What your question really comes down to is, is it clinically significant, that is, does it make a clinical difference? A $2 \%$ difference in ejection fraction in and of itself would not seem to make a difference. However, remember that an absolute increase of $2 \%$ in a patient with a $20 \%$ ejection fraction amounts to a $10 \%$ relative increase in ejection fraction. That may be clinically significant for someone in class III CHF. The only clinical evidence we have is the dramatic improvement in the patient's NYHA class, which becomes very significant. We are attempting to identify measurements in between those two, such as the highly precise oxygen consumption measurements that will be used in phase III to determine whether that small percentage increase in ejection fraction has a clinically significant impact on oxygen consumption. This I think would better answer your question of does that $2 \%$ turn out to be clinically significant, because left ventricular ejection fraction is not the only parameter of heart function that is important. It is one of them, but it is certainly not the only one. Left ventricular stroke work is important. Diastolic function is important. Oxygen consumption is important as well. 